\title{
Multiple relapses in extramedullary localization of acute lymphoblastic leukemia
}

\author{
Kosucu $\mathrm{P}^{1}$, Kul S${ }^{1}$, Gunes $\mathrm{G}^{1}$, Yilmaz $\mathrm{M}^{2}$, Ersoz $\mathrm{S}^{3}$, Ozdemir F² \\ Department of Radiology, Medical School of Karadeniz Technical University, Farabi Hospital, Trabzon, Turkey. \\ polatkosucu@hotmail.com
}

\begin{abstract}
Extramedullary relapses of acute lymphoblastic leukemia in children and young adults are rare and in most cases are usually related to the central nervous system or testes. We describe a case of a 25-yearold man with an acute lymphoblastic leukemia with multiple extramedullary relapses in the testes, gallbladder, breast, parotid gland and eye at various times (Fig. 5, Ref. 21). Full Text in free PDF www.bmj.sk.

Key words: leukemia, MRI, US, testes, gallbladder, breast, parotid gland, eye.
\end{abstract}

Leukemia is a systemic hematological disorder and may affect all organs of the body. The acute lymphoblastic leukemia (ALL) is one of the most aggressive hematological malignancies. About 25-30 \% of patients with ALL experience a recurrence of the disease. The relapse most often involves the bone marrow. Extramedullary ALL relapses are rare and typically involve sequestered sites such as the testes, central nervous system, spleen, liver, kidney, spine and eye $(1,2)$. Extramedullary relapses in the breast, parotid gland and gallbladder are extremely rare (3-10). We describe the clinical presentation and imaging features of a patient with ALL involving extramedullary relapse in the testes, gallbladder, breast, parotid glands, and eye at various times.

\section{Case report}

A 25-year-old-male patient was diagnosed with the acute lymphoblastic leukemia (ALL) in June 2002. A complete remission was obtained after the chemotherapy for remission induction. He was treated with prophylactic radiotherapy to the central nervous system and consolidation and maintenance therapy. The patient was admitted to the hospital for investigation of a palpable mass in the testes in April 2005. Physical examination revealed a bilateral testicular enlargement and hepatosplenomegaly. Hepatosplenomegaly was also confirmed in abdominal ultrasound. There was no focal involvement in the liver or spleen. Scrotal ultrasound revealed a heterogeneous hypoechoic enlarged mass in the right testes and hypoechoic solid masses in the left testes (Figs 1A, B). The ultrasound findings were consistent with leukemic infiltration

${ }^{1}$ Department of Radiology, Medical School of Karadeniz Technical University, Farabi Hospital, Trabzon, Turkey, ${ }^{2}$ Department of Internal Medicine, Medical School of Karadeniz Technical University, Farabi Hospital, Trabzon, Turkey, and ${ }^{3}$ Department of Pathology, Medical School of Karadeniz Technical University, Farabi Hospital, Trabzon, Turkey

Address for correspondence: P. Kosucu, MD, Dept of Radiology, KTU Farabi Hospital, 61080, Trabzon, Turkey.

Phone: +90.4623775674 , Fax: +90.4623252270
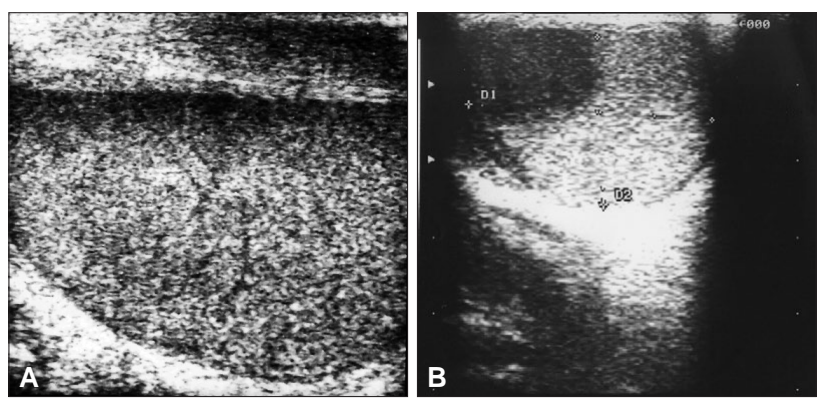

Fig. 1. A) Ultrasound showing longitudinal images of both testicles. The right testes has a highly heterogeneous echo pattern. B) The hypoechoic solid mass in the heterogeneous left testes.
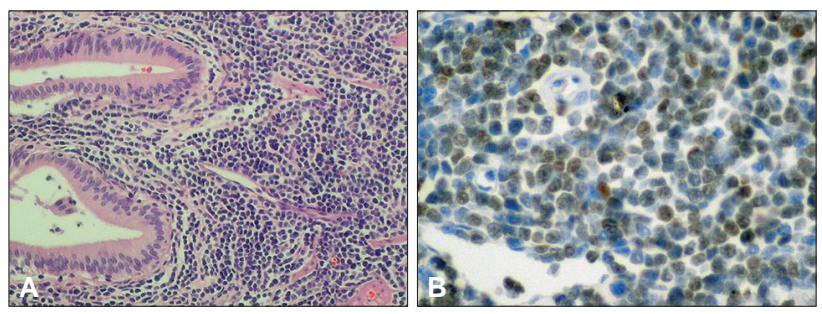

Fig. 2. A) Diffuse atypical lymphoblastic cell infiltration shown in gallbladder mucosa (HEx200). B) Immunohistochemically neoplastic cells positive for Tdt (IHCx400).

of both testicles. Orchidectomy was performed and pathological examination confirmed the diagnosis of ALL infiltration. Bone marrow biopsy showed blast cell infiltration. Second complete remission was obtained with the L-asparagines, vincristine, daunorubicin, prednisolone and cyclophosphamide. Then he was treated by consolidation and maintenance therapy. Hepatosplenomegaly was determined at the physical examination in December 2005. The computed tomography confirmed hepatosplenomegaly and splenomegaly. Splenoctomy and cholecystectomy was performed. Pathological examination revealed a nodular lesion of $2 \mathrm{~cm}$ in diameter located in the fundus of the gallbladder. Microscopic 

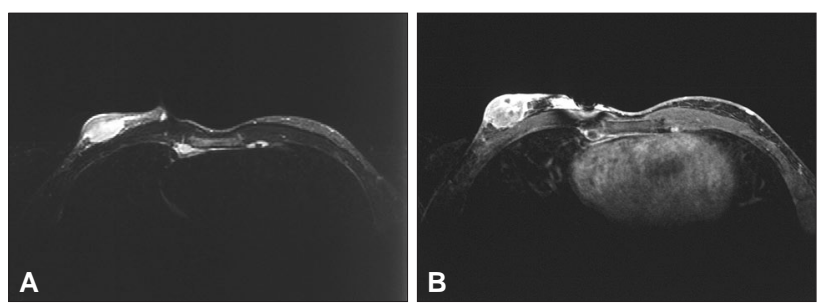

Fig. 3. A) The axial T2-weighted image reveals a hyperintense mass of $4.5 \times 4 \mathrm{~cm}$ in size in the right breast. B) The mass shows a heterogeneous enhancement in an axial Gd-DTPA enhanced fat-sat T1-weighted image.
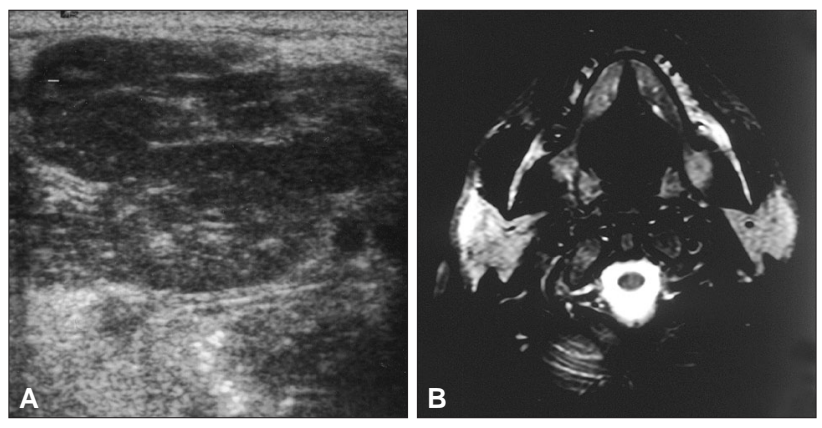

Fig. 4. A) Ultrasound showing a multiple heterogeneous hypoechoic solid mass in the enlargement of the right parotid gland. B) Axial fatsuppressed image showing heterogeneous enlargement of the right parotid gland.
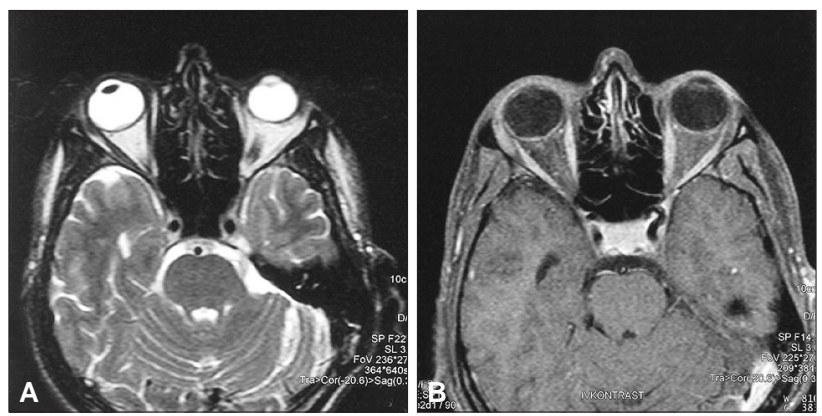

Fig. 5. A) Axial T2-weighted image showing an abnormal convex thickening in the posterior and lateral retina of the right globe (arrows). B) Axial Gd-DTPA enhanced fat-sat T1-weighted image demonstrated one curvilinear enhancement of the posteriorlateral retina (arrows).

examination of the gallbladder revealed a diffuse atypical lymphoblastic cell infiltration. The neoplastic infiltration had thickened the gallbladder wall, especially the fundus. Neoplastic cells were intermediate in size with irregular nuclear contours, fine chromatin networks and scant cytoplasms. Immunohistochemically, neoplastic cells stained positive for Tdt, focal CD3 positivity and negative CD20 (Figs 2A, B). The patient was treated with cytarabine and idarubicin in February 2006. The patient was admitted to hospital for investigation of a mass in his left breast in February 2006. Physical examination revealed a large, non-tender unilateral mass measuring $5 \times 4 \mathrm{~cm}$ in diameter. No axillary lymphadenopathy was found. Mammography revealed a very dense mass. Breast ultrasound revealed a solid, lobulated $5 \times 4 \mathrm{~cm}$ mass. The center of the mass was markedly hypoechoic, with an echogenic rim at the periphery. In the color Doppler ultrasound, the lesion was hypervascular in the center. The appearance of the mass at ultrasound was consistent with malignancy. Breast MRI showed a bilateral multiple solid mass with irregular contours, the largest of which measured $4.5 \times 4 \mathrm{~cm}$ in diameter in the right retroareolar area. The masses were hypointense on T1WI and hyperintense on T2WI, and in addition to homogenous enhancement in the early post-contrast T1WI there was also a diffuse skin thickening on the left (Figs 3A, B). The patient underwent fine-needle aspiration biopsy. Histopathological examination demonstrated leukemic infiltration of the breast. A non-myeloblastive allogenic bone marrow transplant was performed and remission was achieved in May 2006. A control bone marrow aspiration biopsy revealed a fourth relapse in June 2006. He received a donor lymphocyte infusion in September 2006. The patient presented again with vision loss at the perception/projection level and pain in the right eye and progressive bilateral pre-auricular swelling in October 2006. Ultrasound showed an enlargement and heterogeneous echogenity at the parotid glands. There were hypoechoic lymph nodes 5 to 15 $\mathrm{mm}$ in diameter within the bilateral parotid gland (Fig. 4A). MRI of the neck and eyes was performed. This showed a heterogeneous enlargement at the parotid glands on T1WI and T2WI. There were multiple lymph nodes, hypointense on T1WI and hyperintense on T2WI, within the parotid glands (Fig 4B). Ophthalmoscopic examination revealed a retinal elevation close to the optic disk with no signs of retinal hemorrhage. MRI also revealed an abnormal curvilinear retinal thickening with a diameter up to $2 \mathrm{~mm}$ in the right eye. This retinal thickening was hypointense on T1WI and hyperintense on T2WI and showed a diffuse enhancement on T1weighed images with Gd-DTPA enhancement (Figs 5A, B). The patient was admitted to hospital and chemotherapy was initiated. Unfortunately, he died from a multi-organ failure.

\section{Discussion}

Leukemia is a systemic hematological disorder and may affect all organs of the body with diverse clinical manifestations. ALL is the one of the most common and aggressive hematological malignancies. About $25-30 \%$ of patients with ALL experience a recurrence of the disease. Leukemic relapse can present as either hematopoietic or extramedullary relapse of ALL in adults. The bone marrow is the most common site for recurrence. Approximately $25 \%$ of relapses are extramedullary $(1,2)$. The central nervous system and testes, so called sanctuary sites, are the predominant sites of isolated extramedullary disease, while relapses to other localizations are exceedingly rare (11). There are few reports in the literature on relapses affecting the kidneys, eyes, muscles, ovaries, lachrymal gland, abdominal lymph nodes, bones, thoracic wall, mediastinum, orbit, heart or breast $(12,13)$.

The testicle is the most common site of extramedullary leukemia relapse in males (1). It is believed that the testes act as a sanctuary site for leukemia cells during chemotherapy. About one-third of patients with testicular relapse have clinical involvement of both testes. Ultrasound is a typical imaging method of 
choice for evaluating the testicles. Diffuse infiltration, producing diffusely enlarged, hypoechoic testes, is the most frequent presentation. Hypoechoic areas and anechoic masses have been described (14). In our case, ultrasound demonstrated both diffuse and nodular leukemic infiltration in the testes.The right testes was heterogeneously hypoechoic, and there were hypoechoic solid nodular masses in the left testes.

Leukemic infiltration of the gallbladder is unusual, although infiltration of the gastrointestinal tract may also occur. We discovered only 5 case reports describing a relapse of acute lymphoblastic leukemia presenting as leukemic infiltration of the gallbladder wall (3-6, 5). Leukemic infiltration of the gallbladder can present as cholecystitis (3), which was absent in our case. Infiltration of the gallbladder was diagnosed at laparotomy. Histopathological examination showed a nodular infiltration in the fundus and diffuse infiltration in the other wall of the gallbladder.

The breast involvement in acute leukemia is extremely rare and occurs mostly in patients with acute myeloid leukemia. The disease was usually present bilaterally as multiple nodules (7). The ultrasound appearance of acute myeloid leukemia in the breast has been reported as areas of mixed echogenity with or without some acoustic shadowing. The ultrasound appearance of the breast involvement in the acute lymphoblastic leukemia has been reported as a mass with a markedly hypoechoic center and an echogenic rim at the periphery $(7,15,16)$. In our case, ultrasound appearance was similar to that reported by Likaki-Karatza et al (7). The center of the mass was markedly hypoechoic with an echogenic rim at the periphery. The lesion was hypervascular in the center at color Doppler US. The masses were hypointense on T1WI and hyperintense on T2WI, and homogenous enhancement was visible on early post-contrast T1WI.

The ocular lesions are not rare in cases of leukemia and are the third-most-frequent extramedullary location of acute leukemia after the central nervous system and testicles (17). The ocular manifestations are associated with a direct infiltration by leukemia, hemorrhage, ischemia, or toxicity due to various chemotherapeutic agents $(18,19)$. Almost all ocular structures have been found to be involved in patients with various type of leukemia $(19,20)$. The retina is involved in leukemia more often than any other ocular tissue. Leukemic infiltrates and hemorrhages are found at all levels of the retina, but especially in the inner layers with focal destruction. Total retinal detachment may present as an isolated relapse because of large leukemic infiltrates (19). In our case, MRI demonstrated a leukemic involvement of the retina. There was a curvilinear thickening in the retina, hypointense on T1WI and hyperintense on T2WI, and diffuse enhancing on the contrastenhancement T1WI.

The parotid gland is an extremely rare extramedullary site for the recurrence of ALL. Only 4 cases of leukemia involving the parotid glands have been reported $(10,11,21)$. Fukuzawa at al. demonstrated a leukemic infiltration of the parotid gland using the Ga-67 imaging (10). Obama at al. observed a diffuse involvement of leukemic cells in the parotid glands at autopsy (8). To our knowledge, there is only one report of extramedullary relapse in the parotid gland demonstrated by MRI. Rhee at all reported a diffuse enlargement of the parotid gland and hyperintense T2-weighted images (12). In our case, ultrasound and MRI showed a heterogeneous enlargement in the parotid glands. There were hypoechoic lymph nodes in the parotid glands. Intraparotid lymph nodes were hypointense on T1WI and hyperintense on T2WI.

Radiological imaging is very important in the detection of leukemic relapse because several pathologies mimic recurrence. Ultrasound and MRI are the best imaging modalities to evaluate the extramedullary relapse and these play a crucial role in an early detection $(2,17)$. MR imaging is a standard method of evaluating suspicion of relapse because it provides a greater anatomical detail and resolution in the head and neck region and the central nervous system.

\section{References}

1. Henze G, Fengler R, Hartman R et al. Six-year experience with a comprehensive approach to the treatment of recurrent childhood acute lymphoblastic leukemia (ALL-REZ BFM 85). A relapse study of the BFM group. Blood 1991; 78 (1): 1166-1172.

2. Porter RP, Kaste SC. Imaging findings of recurrent acute lymphoblastic leukemia in children and young adults, with emphasis on MRI. Pediatr Radiol 2004; 34 (5): 400-408.

3. Shimizu T, Tajiri T, Akimaru K et al. Cholecystitis caused by infiltration of immature myeloid cells: a case report. J Nippon Med Sch 2006; 73 (2): 97-100.

4. Hurley R, Weisdorf DJ, JessurunJ, Vercellotti GM, Miller WJ. Relapse of acute leukemia presenting as acute cholecystitis following bone marrow transplantation. Bone Marrow Transplant 1992; 10 (4): 387-389.

5. Bloom Sh, Coad JE, Greeno EW, Ashrani AA, hammerschmidt DE. Cholecystitis as the presenting manifestation of acute myeloid leukemia: report of a case. Am J Hematol 2002; 70 (3): 254-256.

6. Buyukasik Y, Kosar A, Demiroglu H, Altinok G, Ozcebe OI, Dundar S. Acalculous acute cholecystitis in leukemia. J Clin Gastroenterol 1998; 27 (2): 146-148.

7. Likaki-Karatza E, Mpadra FA, Karamouzis MV et al. Acute lymphoblastic leukemia relapse in the breast diagnosed with gray-scale and color Doppler sonography. J Clin Ultrasound 2002; 30 (9): 552-556.

8. Obama K, Saito M, Higuchi I, Tara M, Niina K, Osame M. Adult T-cell leukemia predominantly involving exocrine glands. Eur J Haematol 2004; 73 (5): 332-339.

9. Mesa JR, Espinosa E, Losada R, Hernandez C, Martinez G, Hernandez P. Parotid and central nervous system relapse during complete hematologic remission in acute promyelocytic leukemia. Haematologica 1999; 84 (6): 565-566.

10. Fukuzawa J, Akaishi T, Tanaka $\mathbf{H}$ et al. Adult T-cell leukemia with invasion by malignant cells of the parotid glands diagnosed by Ga-67 imaging. Clin Nucl Med 1996; 21 (12): 964-966.

11. Wright TL, Bardy PG, Disney P, Moore S, Horvath N. Isolated cardiac recurrence of acute lymphoblastic leukemia characterized by $\mathrm{t}(11 ; 19)$ two years after unrelated allogeneic bone marrow transplantation. Cancer Genet Cytogenet 2002; 137 (2): 146-149.

12. Huck K, Laws H-J, Meisel R et al. Three cases of renal relapse after allogeneic hematopoietic stem cell transplantation for childhood acute lymphoblastic leukemia. Haematologica 2006; 91 (Suppl 5): ECR07. 
13. Koca E, Goker H, Guven GS et al. Unusual extramedullary recurrences and breast relapse despite hepatic GVHD after allografting in $\mathrm{Ph}+-$ ALL. Hematolog. 2006; 11 (2): 105-107.

14. Phillips G, Kumari-Subaiya S, Sawitsky A. Ultrasonic evaluation of the scrotum in lymphoproliferative disease. J Ultrasound Med1984; 6 (4): 169-175.

15. Bloom SH, Coad JE, Greeno EW, Ashrani AA, Hammerschmidt DE. Cholecystitis as the presenting manifestation of acute myeloid leukemia: report of a case. Am J Hematol 2002; 70 (3): 254-256.

16. Hiorns MP, Murfitt J. Granulocytic sarcoma (chloroma) of the breast: sonographic findings. AJR Am J Roentgenol 1997; 169 (6): 1639-1640.

17. Khoury NJ, Hanna Al-Kass FM, Jaafar HN et al. Bilateral breast involvement in acute myelogenous leukemia. Eur Radiol 200; 10 (6): 1031.
18. Soares MF, Braga FT, Rocha AJ, Lederman HM. Optic nerve infiltration by acute lymphoblastic leukemia: MRI contribution.Pediatr Radiol 2005; 35 (8): 799-802.

19. Paydas S, Soylu MB, Yavuz S et al. Serous retinal detachment in a case with chronic lymphocytic leukemia: no response to systemic and local treatment. Leuk Res 2003; 27 (6): 557-559.

20. Sharma T, Grewal J, Gupta S, Murray PI. Ophthalmic manifestations of acute leukaemias: the ophthalmologist's role. Eye 2004; 18 (7): 663-672.

21. Cardone S, Yen MT, Chevez-Barrios P, Foroozan R, Yen KG. Recurrent acute lymphoblastic leukemia presenting in the lacrimal gland. Ophthal Plast Reconstr Sur. 2006; 22 (1): 56-57.

Received August 23, 2009. Accepted September 20, 2011. 